\title{
Management of Chronic Anal Fissure With A Novel Topical Hemp-Herbal Based Ointment - A Pilot Study
}

\author{
Edward Ram \\ Sheba Medical Center \\ Yaniv Zager \\ Sheba Medical Center \\ Raanan Meyer \\ Sheba Medical Center

\section{Dan Carter} \\ Sheba Medical Center \\ Samia Joubran \\ Sheba Medical Center \\ Nir Horesh ( $\square$ nir_horesh@hotmail.com ) \\ Sheba Medical Center
}

\section{Research Article}

Keywords:

Posted Date: March 7th, 2022

DOI: https://doi.org/10.21203/rs.3.rs-1389433/v1

License: (c) (i) This work is licensed under a Creative Commons Attribution 4.0 International License.

Read Full License 


\section{Abstract \\ Purpose}

Anal fissure (AF) is a common anorectal disease. Although several pharmacological treatments are available, many patients still require surgical interventions. In this study, we aimed to evaluate the efficacy of an ointment based on a multi-functional blend of herbal ingredients including hemp (ProctoFiz) for chronic AF.

\section{Methods}

A single arm questionnaire-based prospective study was conducted in a large tertiary center to evaluate the outcomes of patients suffering from chronic AF treated with topical ProctoFiz.

\section{Results}

Ninety-two patients were included in the study, 54 (58.7\%) were females with a median age of 39 (range 17-78). 32 patients (34.7\%) suffered from recurrent AF before enrolling in the study and 5 patients (5.4\%) underwent previous surgical interventions for AF. Three patients (3.2\%) were lost to follow-up leaving 89 patients for analysis. Eighty patients (89.9\%) reported significant improvement of symptoms after one week using ProctoFiz, and 79 patients reported continued improvement after one month of treatment. The mean Pain Visual Analog Score (VAS) declined by 6.6 points ( 8.9 vs. $2.3 ; 95 \% \mathrm{Cl}-7.20$ to $-5.99, \mathrm{p}<$ 0.0001 ) following one week of treatment, with continuous improvement to a mean of 0.64 after one month. Negative impact on Quality of Life (QoL) significantly decreased from a mean 8.8 to a 0.38 following a month of treatment $(p<0.0001)$, with significant reduction in the number of patients suffering from bleeding following bowel movements $(64.1-2.5 \% ; p=0.0001)$.

\section{Conclusion}

Hemp based topical treatment of AF is feasible and significantly improves AF correlated symptoms.

\section{Introduction}

Anal Fissure is one of the most painful anal disorders and has a negative impact on the patient's quality of life. AF is a linear crack or tear in the squamous epithelium of the lower half of the anal canal, usually extending from below the dentate line to the anal verge. AF is painful because of sensory innervations to this area. Fissures occur in all age groups, with equal prevalence in men and women. Possible causes of AF include infection and traumatic injury to the anal canal such as from passage of hard stool or severe diarrhea ${ }^{1}$, although some studies also suggest that ischemia of the anal canal plays an important role in 
the pathogenesis of $\mathrm{AF}^{14}$. Atypical anal fissures may develop in people with Crohn's disease, sexually transmitted diseases, tuberculosis, post-surgical local trauma, anal intercourse, anal cancer or chemotherapy ${ }^{2}$.

AF can be acute or chronic. Acute AF is a simple rupture in the anoderm existing for less than two months, whereas chronic AF is present for more than 6-8 weeks. The latter is characterized by exposed fibers of internal anal sphincters at the base, hypertrophied proximal anal papilla, and a distal skin tag or sentinel pile.

Both acute and chronic AF are associated with anal pain, spasm, and/or bleeding upon defecation. In the past, surgical treatment of anal fissure was the treatment of choice in order to lower the resting anal pressure. These procedures included anal dilatation and lateral sphincterotomy. Regarding possible complications, anal dilation causes permanent fecal incontinence more often than lateral sphincterotomy ${ }^{3}$.

Currently, initial treatment of AF consists of administration of various medications and local ointments. They include topical agents such as calcium channel blockers and topical nitroglycerin along with laxatives and topical anesthetic creams ${ }^{4}$. These have gained popularity mainly because they enable early intervention and are considerably cheaper compared to surgical intervention ${ }^{19}$. The principal goal is to relieve symptoms by reducing the anal muscle contractility and spasm. This decreases the risk of repeated trauma upon defecation. In addition, injection of botulinum toxin has achieved good results, although repeated injections may be required ${ }^{5}$. Finally, in patients without an adequate response to medical treatment, lateral sphincterotomy of the internal sphincter may be necessary. This procedure achieves high rates of therapeutic success; however, many patients experience significant changes in continence following surgery, ranging from almost $45 \%$ in the immediate post operative period to $1-8 \%$ on long term follow up ${ }^{11-12}$. These outcomes must be taken into consideration when offering surgical intervention ${ }^{6}$.

A proprietary multifunctional herbal-based product for topical application has been developed consisting of natural ingredients, each of which targets a specific pathophysiological mechanism of the disease. For example, to counteract the increase in vascular pressure and ischemia, lowering of resting pressure is achieved by one component with a vasodilatory effect, while other herbal ingredients provide antiinflammatory and analgesic effects. Another ingredient acts as a bio-adhesive, aimed at protecting and healing the fissure wound. The product, ProctoFiz@ (by ESPA Biomedical Ltd, Ramat Hasharon Israel) is currently an over the counter (OTC) cosmetic product, which has been approved as a medical device by the Israeli Ministry of Health. ProctoFiz@ contains the following ingredients: hemp seed oil, calendula, sweet almond, myrtle, arnica and seaweed extract.

We report the clinical outcomes in this prospective questionnaire-based study of patients with chronic AF treated with this novel herbal-based natural product. 


\section{Materials And Methods}

Study participants were adult patients suffering from chronic AF, diagnosed over a period of 18 months by a single senior colorectal surgeon (ER) at an ambulatory colorectal specialty clinic. We included patients presenting with anal pain, which was clinically determined to be associated with a midline (dorsal or ventral) anal fissure for a minimum of one month. All study subjects volunteered to participate in the study and each signed an informed consent. The study was approved by the Institutional Review Board (Helsinki Committee) of Sheba Medical Center. All research was performed in accordance with IRB regulations according to the Declaration of Helsinki. We excluded patients with inconclusive diagnosis of AF or with other chronic conditions, such as inflammatory bowel disease or known immunodeficiencies.

After an initial screening visit, when a baseline questionnaire was given to participants in order to assess their pre-treatment clinical status, patients were treated with ProctoFiz applied to the external anal area three to four times daily for a period of one month. In addition to the topical treatment, all patients were instructed to use laxatives and dietary supplemental fiber.

One week following the initiation of treatment, interviewers (research assistants that had never met the patients nor had they participated in the design of the questionnaire or the analysis of the results) used a telephone questionnaire, which included questions to evaluate the impact of the symptoms on the patient's quality of life using Visual Analog Scale (VAS) for pain. Answers used a scale from " 0 " to " 10 ", with " 10 " signifying the highest negative impact. Additional symptoms were evaluated, including anal bleeding and burning sensation. Patients were telephoned and interviewed a second time, two weeks after the initiation of treatment.

At one month after the screening visit, a third in-person follow-up was scheduled during which a clinical evaluation was performed. Patients with no subjective relief at one month were referred to be evaluated for further medical treatment or surgery.

\section{Product Details}

ProctoFiz is an over-the-counter ointment that consists of a blend of herbal extracts designed to act on the various pathophysiological mechanisms, associated with anal fissure. The product primarily includes hemp seed oil that acts as an analgesic and anti-inflammatory ${ }^{15}$, tocopherol that induces local vasodilatation and various other ingredients such as Arnica Montana flower oil and marine algae extract that have a calming effect on inflamed skin and reduces inflammation ${ }^{16-18}$.

\section{Statistical Analysis}

Statistical analysis was performed using SAS/STAT software (version 9.4 by Statistical Analysis System corporation, North Carolina, United States of America). Data were compared using Fisher's exact test and $\chi^{2}$ to evaluate differences between qualitative variables and using a $t$-test to compare quantitative 
variables. One way ANOVA was used to determine statistically significant differences between the means of three or more groups. A $P$ value of $<0.05$ based on a two tailed analysis was considered significant.

\section{Results}

Out of 102 patients found eligible to participate in the study, 10 patients declined to participate leaving ninety-two patients eligible to participate in the study, 54 (58.7\%) of which were female. Median age was 39 years (range 17-78). Eighty-nine (96.8\%) patients suffered from constipation with anal pain and difficulties with defecation. Mean duration from the beginning of symptoms to the screening visit was 20 weeks (range 4-96 weeks). Sixty patients (65.3\%) were diagnosed with AF at the initial visit or were referred by a family practitioner to our proctology clinic with complaints suggesting AF. Thirty-two (34.7\%) patients suffered from recurrent symptomatic events before enrolling in the study and were previously treated with topical CCB's and topical analgesics. None of the patients were previously treated with Botox injections and 5 (5.4\%) patients had previously underwent lateral sphincterotomy for AF. Three (3.2\%) patients were lost to follow up (two did not answer the telephone and one did not appear for the follow up visit), leaving 89 patients for final analysis.

After one week of treatment with ProctoFiz@ 80 (89.9\%) patients reported a significant improvement of symptoms: a significant reduction in pain and/or anal bleeding. After one month of treatment, 79 patients reported continued improvement. Regarding side effects, two (2.2\%) patients complained of headaches one week after initiation of treatment, which resolved the following week. Seven patients did not report any improvement in their symptoms. Six patients were treated with topical Nifedipine. Of these patients who were treated with Nifedipine, three patients were eventually referred for surgical intervention (lateral sphincterotomy) due to failure of medical therapy.

Symptomatic improvement following one week of treatment was demonstrated by a statistically significant decrease in the mean VAS pain score: from 8.9 to $2.3-$ a total of 6.6 points $(95 \% \mathrm{Cl}-7.20$ to $-5.99, p<0.0001)$. At the one month follow up visit, continued improvement in anal pain was seen with a mean VAS Score of 0.64 (Fig. 1). Furthermore, in patient reports after one week of treatment about the length of time of pain and/or discomfort following a bowel movement, a significant decrease was noted, from a mean of 6.2 to 1.34 hours. Patients reported a decrease in duration of pain after a bowel movement after using the ointment for 2 weeks, with a slight increase in duration of pain between two weeks and one month (mean 0.45 to 1.01 hours) (Fig. 2). Overall, patients reported a decrease in the negative impact of their symptoms on their Quality of Life (QoL), with a statistically significant decrease from a mean of 8.8 to 0.38 following one month of treatment $(p<0.0001)$ (Fig. 3).

Fifty-nine (64.1\%) patients suffered from anal bleeding following bowel movements prior to the initiation of treatment. After one week of treatment, 11 (12.4\%) patients continued to suffer from bleeding associated with bowel movements. At one month of follow-up, only 2 patients (2.2\%) suffered from anal bleeding (Fig. 4). In addition, the number of patients who complained of anal burning sensation following 
bowel movement decreased from $82(89.1 \%)$ patients at initial screening to $27(29.4 \%)$ patients at one week and 12 patients (13.1\%) at one month of treatment. (Fig. 5).

\section{Discussion}

$\mathrm{AF}$ is one of the most common causes of anorectal pain and discomfort, often accompanied by a burning sensation and bleeding after defecation. In many patients, the initial tear in the anoderm causes a vicious cycle of persistent pain, as many of these patients try to defer defecation due to the associated pain $^{7}$. The mainstay of treatment of AF aims to break this vicious cycle, to ease defecation by reducing anal sphincter spasm and pressure in the area, thus preventing additional trauma to the anal mucosa and promoting healing of the fissure ${ }^{8}$.

Therapeutic options for patients suffering from chronic AF are divided into pharmacological treatments and surgical intervention. Although it is estimated that around $35 \%$ of patients will spontaneously heal for a brief period, recurrence is quite common ${ }^{13}$. Surgical intervention, although proven to be the most effective method of treatment, should be offered to patients only after pharmacological treatment has failed, because it carries a significantly higher risk of anal incontinence, severely impacting the patient's quality of life. For this reason, patients generally are first given a trial of local treatment with ointments containing topical nifedipine or nitroglycerine, both vasodilators that reduce the muscle spasm of the anal sphincter ${ }^{9,10}$. However, side effects of these medication are quite common and include hypotension, headaches and dizziness.

In this study, we report the initial results of a single arm pilot study of patients with chronic AF treated with a novel herbal-based natural ointment, ProctoFiz@ $\mathbb{0}$. Although our study lacks a control group and has a fairly limited time period for follow up, the results are encouraging as almost $90 \%$ of patients reported significant improvement or complete resolution of their symptoms after one month of treatment. This significant improvement in the patients' symptoms, is similar to reported outcomes in standard topical agents including nitroglycerine and calcium channel blockers standing at $48-92 \%{ }^{20}$. Of particular note, is that the maximal or most significant effect of using the ointment in most patients was observed after only one week of treatment and that the rate of side effects is considerably low in our study.

The positive results in this study were achieved using ProctoFiz as the therapeutic agent in conjunction with standard directives regarding stool softeners and dietary advice. We believe that the multi-functional composition of this product effectively addressed the underlying causes of AF. Standard conventional medications usually target one mechanism for healing: lowering anal pressure through vasodilation by blocking the calcium channel. ProctoFiz is a product with a mix of herbal components that advantageously acts with multiple mechanisms to achieve superior efficacy for vasodilation, lubrication, wound protection, pain relief, and anti-inflammatory effect.

The main limitation of our study was the single arm design with the lack of a control group. Although our results are encouraging, especially considering the fast response to treatment in the majority of patients, 
the lack of comparison to patients treated with placebo or to standard treatments obligates us to take these results with necessary caution. Another caveat of our study is the fairly short follow up period. However, in our experience, most patients with a fissure are not followed up for a long period and we believe that a shorter follow up represents real life experience. The high success rate of eliminating symptoms suggests that topical application of the novel herbal-based product, ProctoFiz, can be considered as an effective and safe non-surgical method for short-term management of chronic AF. In addition, we believe that this alternative should be considered in patients who develop significant side effects from standard treatments for AF. Further comparative studies are needed to determine whether this treatment could be considered as a first line treatment for relief of symptoms and healing of AF.

\section{Declarations}

Conflict of Interest: The authors declare that they have no conflict of interest.

Funding: None

Data availability - The datasets generated during the current study are not publicly available but will be available from the corresponding author on reasonable request.

\section{References}

1. Ram E, Alper D, Stein GY, et al. Internal Anal Sphincter Function Following Lateral Internal Sphincterotomy for Anal Fissure, A Long-term Manometric Study. Ann Surg 2005;242(2):208-211.

2. Zaghiyan K, Fleshner P. Anal fissure. Clin Colon Rectal Surg 2011; 24:22-30.

3. Ram E, Vishne T, Lerner I, Dreznik Z. Anal dilatation versus left lateral sphincterotomy for chronic anal fissure: a prospective randomized study. Techniques in Coloproctology, December 2007.

4. Madalinski MH. Identifying the best therapy for chronic anal fissure. World J Gastrointest Pharmacol Ther. 2011;2(2):9-16.

5. Maria G, Brisinda G, Bentivoglio AR, et al. Botulinum toxin injections in the internal anal sphincter for the treatment of chronic anal fissure: long-term results after two different dosage regimens. Annals of Surgery. 1998;228(5):664-669.

6. Littlejohn DRG, Newstead GL. Tailored lateral sphincterotomy for anal fissure. Diseases of the Colon \& Rectum. 1997;40(12):1439-1442.

7. Schlichtemeier S, Engel A. Anal fissure. Aust Prescr. 2016;39(1):14-17.

8. Braun J, Raguse T. Pathophysiologic role of the internal anal sphincter in chronic anal fissure. Zeitschrift fur Gastroenterologie. 1985;23(10):565-572.

9. Lund J, Nyström P-O, Coremans G, et al. An evidence-based treatment algorithm for anal fissure. Techniques in coloproctology. 2006;10(3):177.

10. Steele S, Madoff R. Systematic review: the treatment of anal fissure. Alimentary pharmacology \& therapeutics. 2006;24(2):247-257. 
11. Nyam DC, Pemberton JH. Long-term results of lateral internal sphincterotomy for chronic anal fissure with particular reference to incidence of fecal incontinence. Dis Colon Rectum. 1999 Oct;42(10):1306-10.

12. Elsebae MM. A study of fecal incontinence in patients with chronic anal fissure: prospective, randomized, controlled trial of the extent of internal anal sphincter division during lateral sphincterotomy. World J Surg. 2007 Oct;31(10):2052-7.

13. Nelson RL. Anal fissure (chronic). BMJ Clin Evid. 2014 Nov 12;2014:0407.

14. Beaty JS, Shashidharan M. Anal Fissure. Clin Colon Rectal Surg. 2016;29(1):30-37.

15. Mlost J, Bryk M, Starowicz K. Cannabidiol for Pain Treatment: Focus on Pharmacology and Mechanism of Action. Int J Mol Sci. 2020;21(22):8870.

16. Thiele JJ, Ekanayake-Mudiyanselage S. Vitamin E in human skin: organ-specific physiology and considerations for its use in dermatology. Mol Aspects Med. 2007 Oct-Dec;28(5-6):646-67

17. Klaas CA, Wagner G, Laufer S, Sosa S, Della Loggia R, Bomme U, Pahl HL, Merfort I. Studies on the anti-inflammatory activity of phytopharmaceuticals prepared from Arnica flowers. Planta Med. 2002 May;68(5):385-91

18. Simpi CC, Nagathan CV, Karajgi SR, Kalyane NV. Evaluation of marine brown algae Sargassum ilicifolium extract for analgesic and anti-inflammatory activity. Pharmacognosy Res. 2013 Jul;5(3):146-9.

19. Nelson RL, Thomas K, Morgan J, Jones A. Non surgical therapy for anal fissure. Cochrane Database Syst Rev. 2012 Feb 15;2012(2):CD003431

20. Jin JZ, Hardy MO, Unasa H, Mauiliu-Wallis M, Weston M, Connolly A, Singh PP, Hill AG. A systematic review and meta-analysis of the efficacy of topical sphincterotomy treatments for anal fissure. Int $J$ Colorectal Dis. 2021 Oct 4

\section{Figures}




\section{Pain}

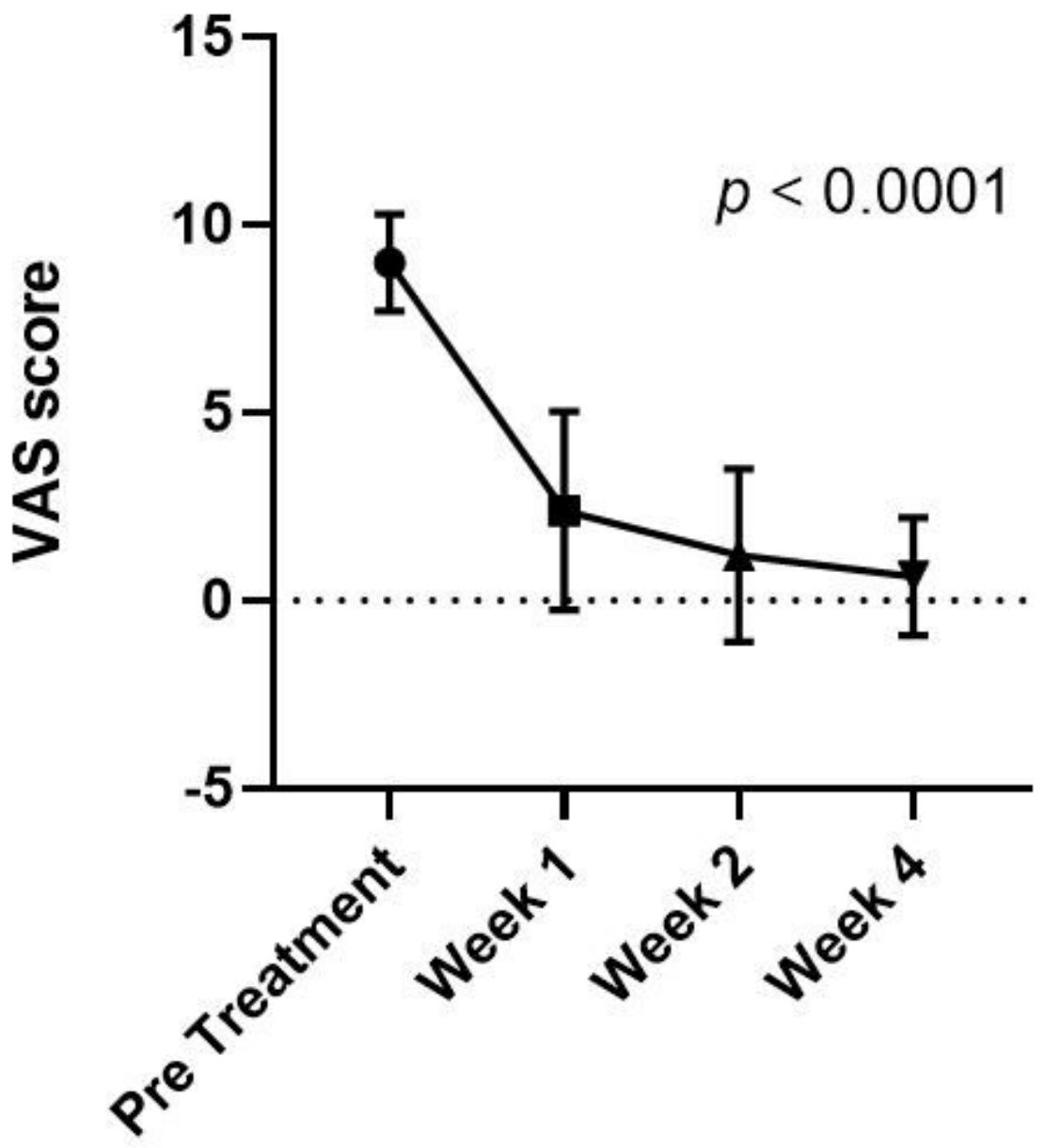

Time

Figure 1

Visual Analog Score (VAS) assessment of pain from anal fissure, prior to treatment, and at follow-up at one, two and four weeks. 


\section{Pain after Bowel Movement}

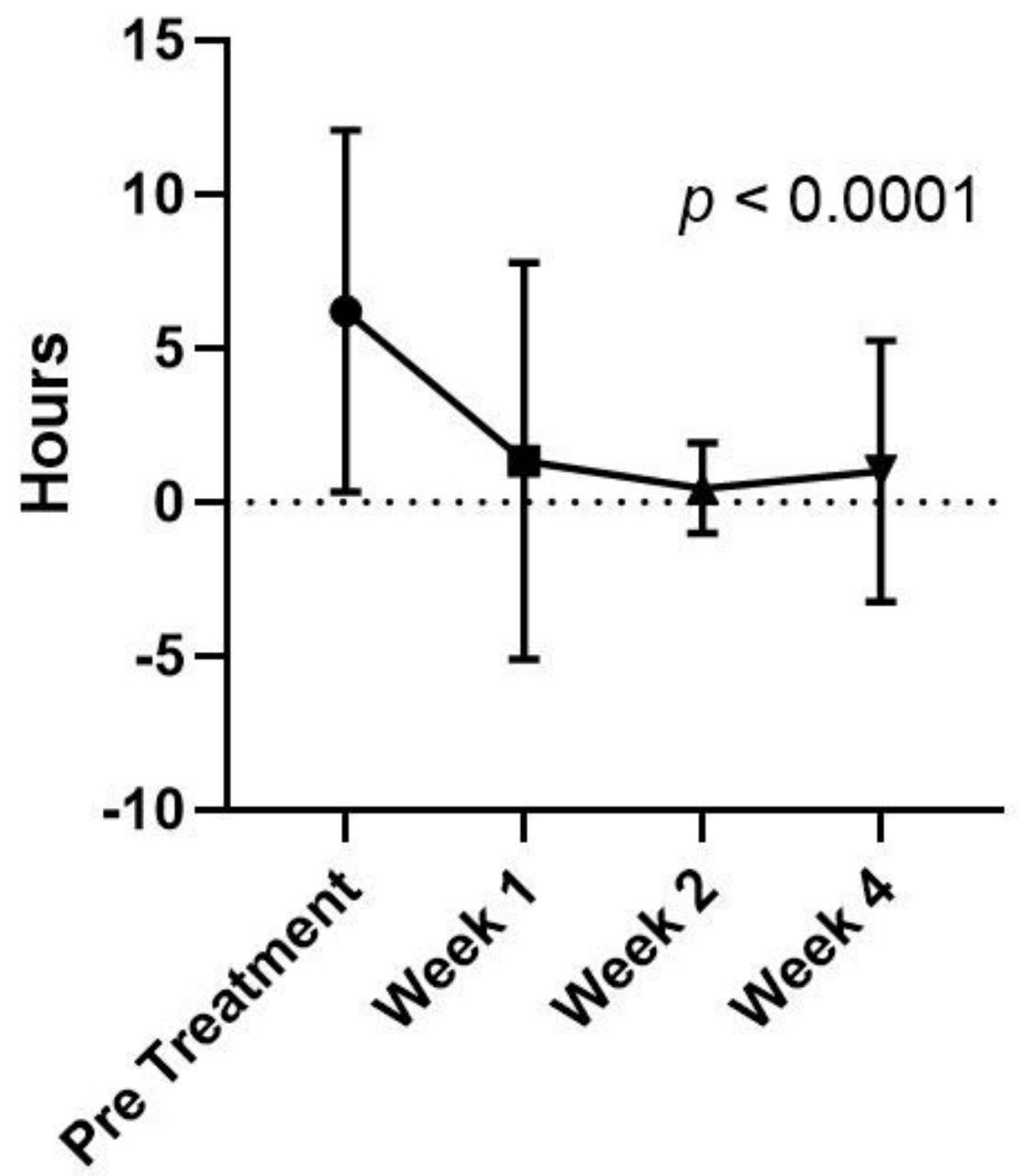

Figure 2

Assessment of the duration of pain following bowel movement in patients with anal fissure, prior to treatment, and at follow-up at one, two and four weeks. 


\section{Impact on Quality of Life}

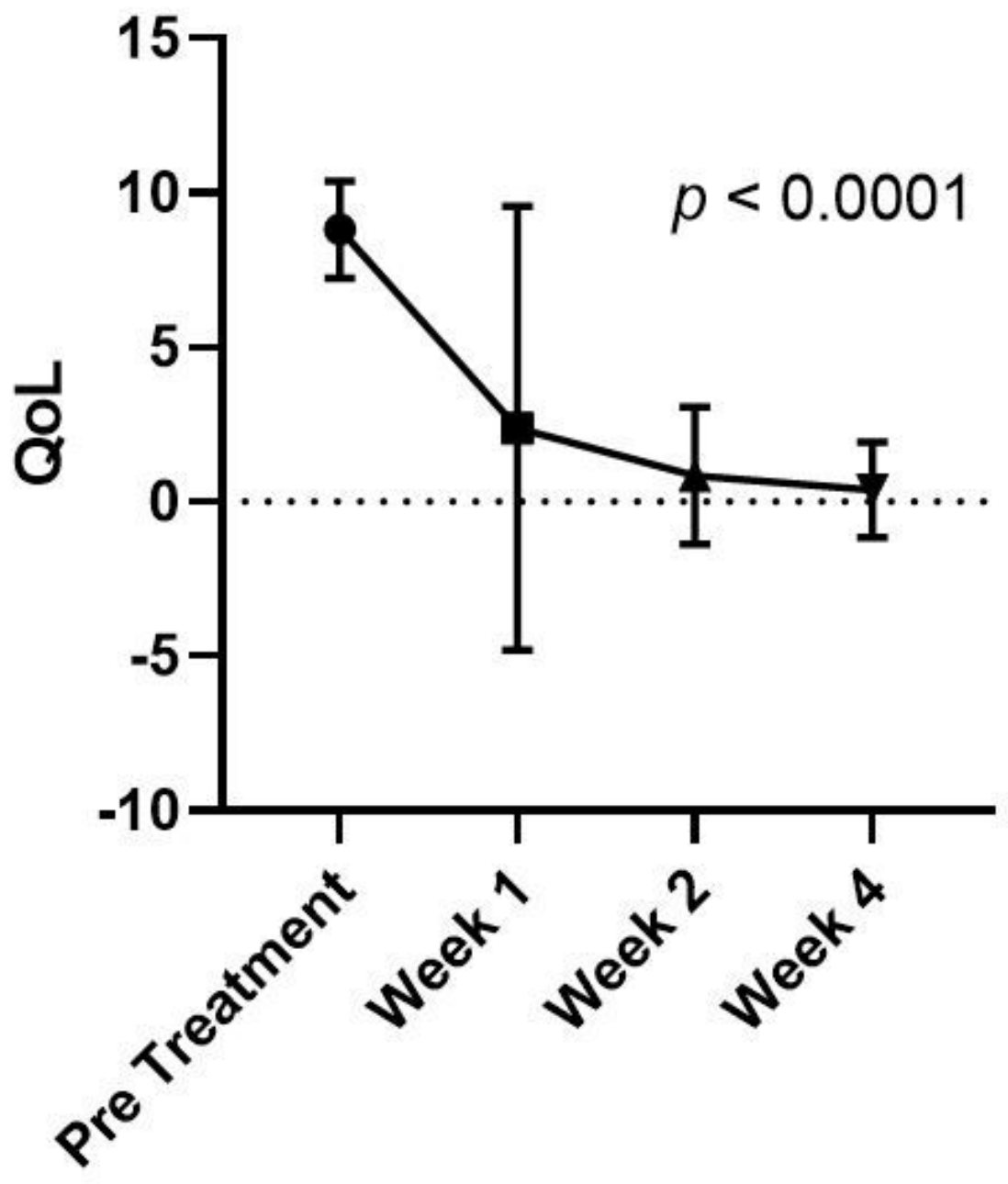

Time

Figure 3

Evaluation of the impact by symptoms on Quality of Life (QoL), in patients with anal fissure prior to treatment, and at follow-up of one, two and four weeks. 


\section{Bleeding after Bowel Movement}

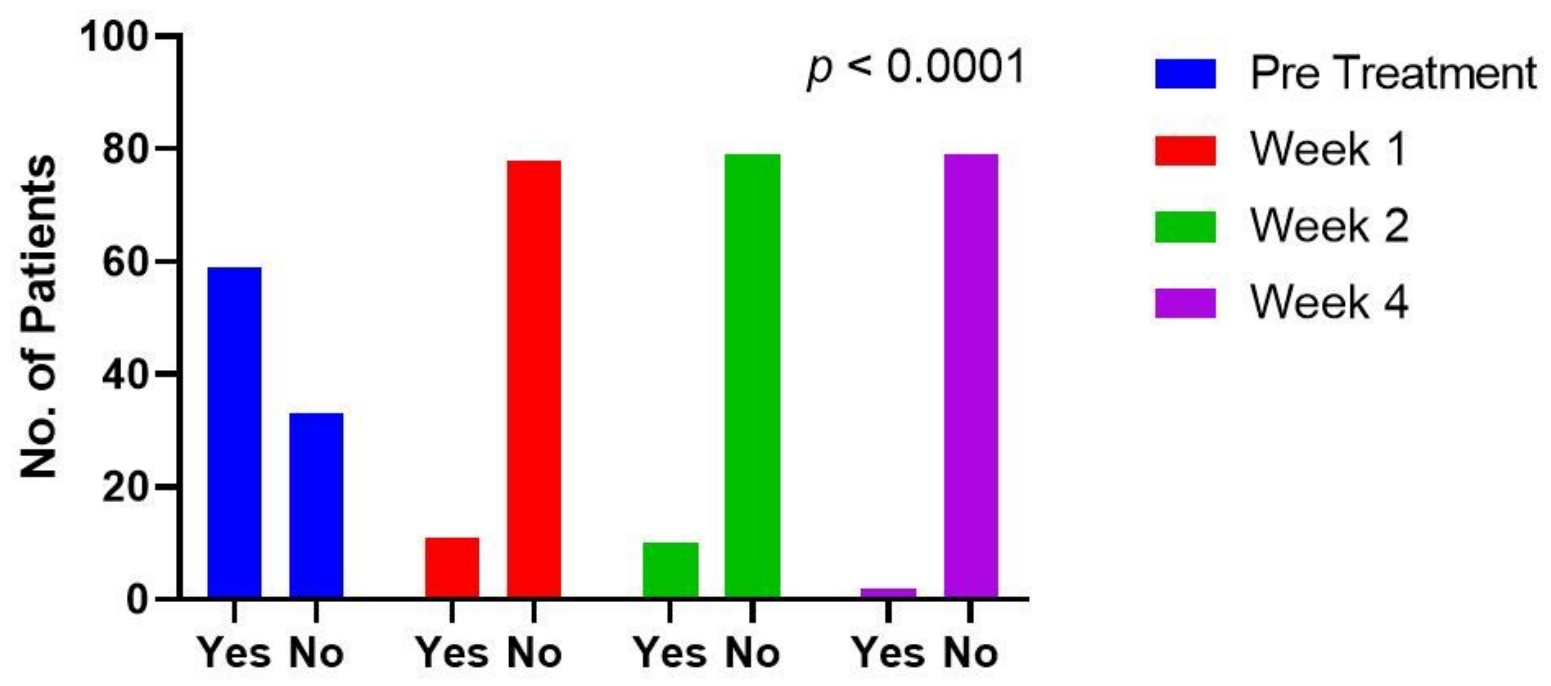

Figure 4

Number of patients with anal fissure suffering from anal bleeding, related to defecation prior to the initiation of treatment, and at follow-up of one, two and four weeks.

\section{Burning Sensation}

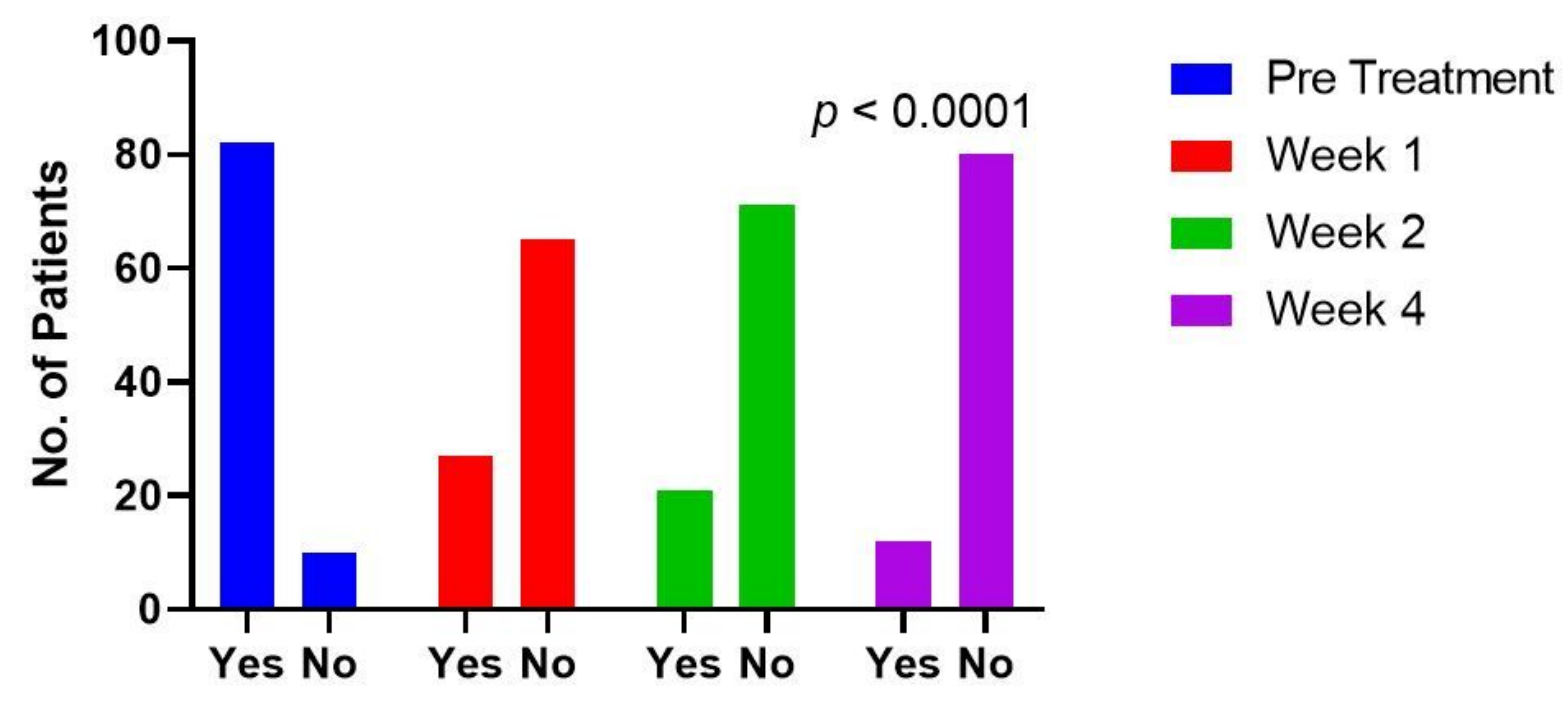




\section{Figure 5}

Number of patients with anal fissure suffering from burning sensation, prior to treatment, and at followup of one, two and four weeks. 